\title{
Efficacy and Safety of Ningmitai Capsules in Patients with Chronic Epididymitis: A Prospective, Parallel Randomized Controlled Clinical Trial
}

\author{
Zhang Jing, ${ }^{1}$ Guan Liying, ${ }^{2}$ Wang Zhenqing, ${ }^{3}$ Zhang Hui, ${ }^{3}$ Liu Shuai, ${ }^{3}$ Sun Dingqi, ${ }^{3}$ \\ Fu Qiang $\left(\mathbb{1},{ }^{3}\right.$ and Zhang Keqin $\mathbb{1}^{3}$ \\ ${ }^{1}$ Department of Nephrology, Shandong Provincial Hospital Affiliated to Shandong First Medical University, Jinan 250021, \\ Shandong, China \\ ${ }^{2}$ Physical Examination Center, Shandong Provincial Hospital Affiliated to Shandong First Medical University, Jinan 250021, \\ Shandong, China \\ ${ }^{3}$ Department of Urology, Shandong Provincial Hospital Affiliated to Shandong First Medical University, Jinan 250021, \\ Shandong, China
}

Correspondence should be addressed to Fu Qiang; qiangfu68@163.com and Zhang Keqin; 270638805@qq.com

Received 8 June 2020; Revised 4 March 2021; Accepted 16 March 2021; Published 29 March 2021

Academic Editor: Adolfo Andrade-Cetto

Copyright (c) 2021 Zhang Jing et al. This is an open access article distributed under the Creative Commons Attribution License, which permits unrestricted use, distribution, and reproduction in any medium, provided the original work is properly cited.

Objectives. To evaluate the efficacy and safety of Ningmitai (NMT) capsules in patients with chronic epididymitis. Methods. This prospective randomized controlled trial included 112 patients diagnosed with chronic epididymitis. The patients were randomized $(1: 1: 1)$ to receive levofloxacin (LVX), NMT, or NMT combined with LVX for 4 weeks. The patients were followed up at 2 and 4 weeks after initiation of treatment and were evaluated in terms of Chronic Epididymitis Symptom Index (CESI) scores, epididymal nodules, and safety parameters. The primary endpoints were the CESI scores at the end of 2 and 4 weeks of treatment. The secondary endpoints included the mean epididymal nodule diameter and the clinical efficacy rate. Safety was evaluated by hepatorenal function tests and adverse event reports during the trial. Results. After 2 weeks of treatment, the CESI score of the NMT group was significantly lower than that of the LVX group $(P<0.05)$. In addition, the clinical efficacy rate of the NMT group was significantly higher than that of the LVX group (55\% vs. $8.33 \%, P<0.0001$ ), indicating that NMT has a rapid effect on chronic epididymitis. After 4 weeks of treatment, there was no significant difference in CESI scores or clinical efficacy rates between the two monotherapy regimens $(P>0.05)$; however, the mean diameter of epididymal nodules was significantly smaller in the NMT group than in the LVX group $(P<0.0001)$. Moreover, after 4 weeks of treatment, the patients in the LVX + NMT group, which had a clinical efficacy rate of $97.22 \%$, had lower CESI scores (both $P<0.01$ ) and a smaller epididymal nodule diameter (vs. LVX, $P<0.0001$; vs. NMT, $P<0.05$ ) than those in the other two groups. No adverse events or abnormal hepatorenal function were found during the study. Conclusion. NMT significantly improved CESI scores and epididymal nodule diameter in patients with chronic epididymitis. The combination of NMT and LVX provides a much better effect than monotherapy, and this treatment regimen was well tolerated.

\section{Introduction}

Chronic epididymitis is a common disease of the male reproductive system. The aetiology of chronic epididymitis is complicated and varied, and the pathogenesis of the disease is usually identified as secondary to prostatitis or urinary tract infections [1]. There is no standard therapy for chronic epididymitis, and the administration of antibiotics remains the most common treatment [2].
Increasing evidence has demonstrated that complementary and alternative medicine is important and effective in the management of different chronic diseases, including urinary retention [3], degenerative knee osteoarthritis [4], gastroesophageal reflux disease [5], chronic idiopathic urticaria [6], and shigellosis [7].

Ningmitai (NMT) capsules, a formulated Chinese medicine, are composed of Touhualiao (Polygonum capitatum Buch.-Ham. ex D. Don), Baimaogen (Imperata 
cylindrica Beauv. var. major (Nees) C. E. Hubb.), Dafengteng (Cocculus orbiculatus (L.) DC.), Sankezhen (Berberis soulieana Schneid., Berberis wilsonae Hemsl., Berberis poiretii Schneid., and Berberis vernae Schneid.), Xianhecao (Agrimonia pilosa Ledeb.), Mufurongye ( $\mathrm{Hi}$ biscus mutabilis L.), and Lianqiao (Forsythia suspensa (Thunb.) Vahl) [8]. These herbal components contribute to multiple pharmacological effects of NMT, including its antibacterial, anti-inflammatory, and analgesic actions, among others $[9,10]$. NMT has been widely used for decades in the treatment of genitourinary diseases, including chronic prostatitis and lower urinary tract infection [11-13] (approval number from the National Medical Products Administration of China: Z20025442). A previous study indicated that NMT in combination with antibiotics had a beneficial clinical effect in the treatment of acute epididymitis [14]. However, the efficacy of NMT for chronic epididymitis remains unclear. Therefore, a prospective randomized study was conducted to assess the efficacy and safety of NMT alone or in combination with antibiotics in patients with chronic epididymitis.

\section{Methods}

2.1. Study Design. This was a prospective, parallel randomized controlled study conducted at the Department of Urology, Shandong Provincial Hospital. Patients diagnosed with chronic epididymitis from June 2017 to March 2019 were screened, and the eligible patients were randomly $(1: 1: 1)$ divided into three groups-a levofloxacin (LVX) group, an NMT group, and a combined (LVX + NMT) group- according to a random code sequence generated by SPSS 18.0 software. In the LVX group, patients received LVX (Daiichi Sankyo Pharmaceutical Co., Ltd. Beijing, China) $0.5 \mathrm{~g}$ q.d. in the morning for 4 weeks. In the NMT group, patients received NMT (Guiyang Xin Tian Pharmaceutical Co., Ltd., China) $0.38 \mathrm{~g} \times 4$ capsules t.i.d. for 4 weeks. Each NMT capsule contains no less than $1 \mathrm{mg}$ gallic acid according to the national drug standard WS-10348- (ZD-0348) 2002-2012Z and the Chinese pharmacopoeia. In the LVX + NMT group, patients received LVX ( 0.5 g q.d. in the morning) plus NMT $(0.38 \mathrm{~g} \times 4$ capsules t.i.d.) for 4 weeks.

This study was conducted under Good Clinical Practice requirements and approved by the Ethics Committee of Shandong Provincial Hospital affiliated with Shandong University, China (No. 2017-054). All patients provided written informed consent before any study procedures.

2.2. Patients. Patients receiving LVX and/or NMT were required to meet the following criteria to be included in this study [15]: (1) age ranging from 18 to 60 years; (2) a history of acute epididymitis or chronic prostatitis; (3) discomfort such as swelling and/or pain that occurred in one or both epididymides or part of the scrotum; (4) mild, intermittent discomfort to severe, persistent pain, and other symptoms of varying degrees; (5) unilateral or bilateral enlargement of the epididymis, with epididymal nodules; (6) local nodular enlargement, irregular margins with low or slightly strong echoes, and abundant blood flow signal on ultrasonography; and (7) willingness to participate in and complete this study.

The exclusion criteria were as follows: (1) suspected or confirmed epididymal tuberculosis; (2) epididymal or testicular tumours; (3) acute testicular epididymitis or an acute episode of chronic epididymitis; (4) severe heart, liver, kidney, or haematopoietic diseases; (5) use of similar drugs within two weeks before treatment; and (6) mental disorders or severe mental disorders.

2.3. Efficacy Assessments. To evaluate the severity of chronic epididymitis, Nickel [16] proposed the Chronic Epididymitis Symptom Index (CESI), which includes 2 domains: a chronic epididymitis pain subscore (range 0 15) and a chronic epididymitis quality-of-life impact domain (range 0 12). Because it accurately assesses the severity of chronic epididymitis, the CESI has been widely recognized and used in clinical practice and clinical research for baseline evaluation and follow-up of patients with chronic epididymitis [17-19].

The primary endpoints in this study were the mean CESI scores after 2 and 4 weeks of treatment. The secondary endpoints were the mean epididymal nodule diameter and the clinical efficacy rate. The clinical efficacy criteria were defined as follows. Effective: the CESI pain score decreased to less than 6 points or reduced by at least 3 points and symptoms improved. Ineffective: the CESI pain score was greater than 5 points or decreased by less than 3 points, and symptoms were unimproved or even worsened.

In our study, the CESI score was determined in all patients before treatment and at the end of 2 and 4 weeks of treatment. Before treatment and after 4 weeks of treatment, all patients underwent colour Doppler ultrasonography. The patients were placed in the supine position, and the penis was placed against the abdominal wall. The scrotum was fully exposed. Three diameters of epididymal nodule were measured by three experienced ultrasound doctors separately.

2.4. Safety Assessments. Safety assessment was based on adverse event reports and hepatorenal function tests, including the examination of alanine aminotransferase (ALT), blood urea nitrogen (BUN), and creatinine (CREA) values.

2.5. Statistical Analyses. All relevant data were collected and statistically analysed. The measurement data were expressed as the mean $\pm \mathrm{SD}$. The baseline characteristics of the study groups were analysed by one-way analysis of variance if the multiple sets of variables met the assumptions of normality and homogeneity of variance; otherwise, the Kruskal-Wallis test was used. The changes over time in the groups were analysed by two-way repeated measure analysis of variance, and the differences were then calculated by Tukey's multiple comparison test. Categorical data are reported as percentages and were compared using the $\chi^{2}$ test. Statistical analysis was performed using GraphPad Prism version 8.0.2 (GraphPad Software, Inc., La Jolla, CA, USA). $P<0.05$ was considered statistically significant. 


\section{Results}

3.1. Baseline Characteristics. A total of 122 patients were enrolled in the study and randomly allocated at a ratio of 1 : $1: 1$ to receive three different treatments. Ten patients withdrew during the treatment and the follow-up period; thus, 112 patients were available for the efficacy evaluation (Figure 1).

The baseline demographic and clinical characteristics of the patients are given in Table 1. There were no significant differences with respect to age, baseline CESI score, or mean diameter of epididymal nodules (MDE) among the LVX, NMT, and LVX + NMT groups $(P>0.05)$.

3.2. Therapeutic Effects. The posttreatment CESI scores of the three groups are summarized in Table 2. After 2 or 4 weeks of treatment, the CESI scores of all the groups had decreased significantly compared with the baseline score $(P<0.05)$, and the effect was further enhanced as the treatment time was extended ( 4 -week vs. 2 -week, $P<0.05$ ). In addition, after 2 weeks of treatment, the CESI score of the NMT group was significantly lower than that of the LVX group (NMT group vs. LVX group, $P<0.05$ ) and further decreased to $12.53 \pm 2.64$ in the combination group (LVX + NMT group vs. LVX group, $P<0.0001)$. After 4 weeks of treatment, the CESI score of the LVX + NMT group was $8.64 \pm 2.15$, which was lower than that of either the LVX or NMT group (both $P<0.01$ ).

As shown in Figure 2, the ultrasound results showed that after 4 weeks of treatment, the MDE was significantly smaller in the NMT group and LVX + NMT group than in the LVX group $(P<0.0001)$, which indicated that NMT, with or without LVX, may be superior to LVX alone in reducing the size of epididymal nodules.

The clinical efficacy rates after 2 or 4 weeks of treatment are given in Table 3. After 2 weeks of treatment, the clinical efficacy rates of the NMT group and the LVX + NMT group were significantly higher than those of the LVX group (NMT group vs. LVX group, 55\% vs. $8.33 \%, P<0.0001$; LVX + NMT group vs. LVX group, $61.11 \%$ vs. $8.33 \%, P<0.0001)$. After 4 weeks of treatment, there was a significant increase in the clinical efficacy rate of each group compared with 2 weeks of treatment (all $P<0.05$ ). In particular, the effective percentage of clinical response in the LVX + NMT group was $97.22 \%$, which was the highest rate in any group.

3.3. Safety Evaluation. The evaluation of hepatorenal function after 4 weeks of treatment, including ALT, BUN, and CREA, is given in Table 4. All data were within the reference value range, and no abnormal hepatorenal function was found in any of the three groups following treatment.

\section{Discussion}

The aetiology of epididymitis is complex and diverse, but its pathogenesis is usually considered to occur after prostatitis or urinary tract infection; as the disease develops, it seriously affects patients' mental health and quality of life [15]. At present, the treatment of epididymitis with extracts has not been determined. Antibiotics (74\%) and anti-inflammatory drugs (36\%) are still the main clinical treatments [16]. In addition, some chronic epididymitis patients choose epididymectomy in response to lingering pain, which is usually caused by varicocele leading to a decrease in local immune activity [20]. However, a previous study indicated that patients with chronic pain from epididymitis have only a $55 \%$ chance of improvement [21]. Therefore, a combination of modalities is considered beneficial for patients with chronic epididymitis [22].

In recent years, traditional Chinese medicine (TCM), as a common complementary and alternative medicine therapy, has been used in the treatment of chronic epididymitis [23]. NMT is an effective TCM product based on its multicomponent, multitarget, and multimechanism therapeutic philosophy. NMT contains certain herbals and effective constituents such as gallic acid, quercetin, rutin, berberine, and luteolin, which play important anti-inflammatory, antioxidative, analgesic, and antibacterial roles [21, 24-27]. Since the symptoms targeted by NMT are consistent with those of chronic epididymitis, we suspected that NMT might be effective against chronic epididymitis.

This was the first parallel randomized controlled clinical study to evaluate the efficacy and safety of NMT capsules in chronic epididymitis. LVX, an antibody that is commonly used in the clinic for chronic epididymitis, was chosen as a control drug. In this study, after 2 weeks of treatment, the CESI score of the NMT group was significantly lower than that of the LVX group $(P<0.05)$, and the clinical efficacy rate was significantly higher than that of the LVX group $(P<0.0001)$, indicating that NMT has rapid efficacy in chronic epididymitis. After 4 weeks of treatment, the outcomes including the CESI score, MDE, and clinical efficacy rate were all further improved in the NMT group, and the MDE of the NMT group was remarkably smaller than that of the LVX group $(P<0.0001)$. A recent research revealed that NMT has anti-inflammatory and antioxidative effects in an autoimmune prostatitis rat model and improves chronic pain by decreasing substance $\mathrm{P}$ in the dorsal root ganglia [10]. In addition, NMT can restore the tension of posterior urethral and bladder neck smooth muscle, reducing urine reflux and the risk of retrograde infection [28]. These pharmacological effects may be the main reason for the effectiveness of NMT against chronic epididymitis. Moreover, NMT combined with LVX further reduced CESI scores and epididymal nodule, with a response rate up to $97.22 \%$, suggesting that the combination regimen could benefit more patients than either monotherapy regimen. Previous studies reported that NMT can inhibit the proliferation of Staphylococcus sp. and Escherichia coli and the formation of their biofilms $[9,29]$. It is an important evidence that NMT combination therapy is superior to single-drug regimen by enhancing the sensitivity of bacteria to antibiotics and exerting a synergistic role. No adverse events or hepatorenal function indicators were observed during the study.

The present study has some limitations. The sample size was relatively small in this study, as it was designed to preliminarily observe the efficacy of NMT alone or combined 


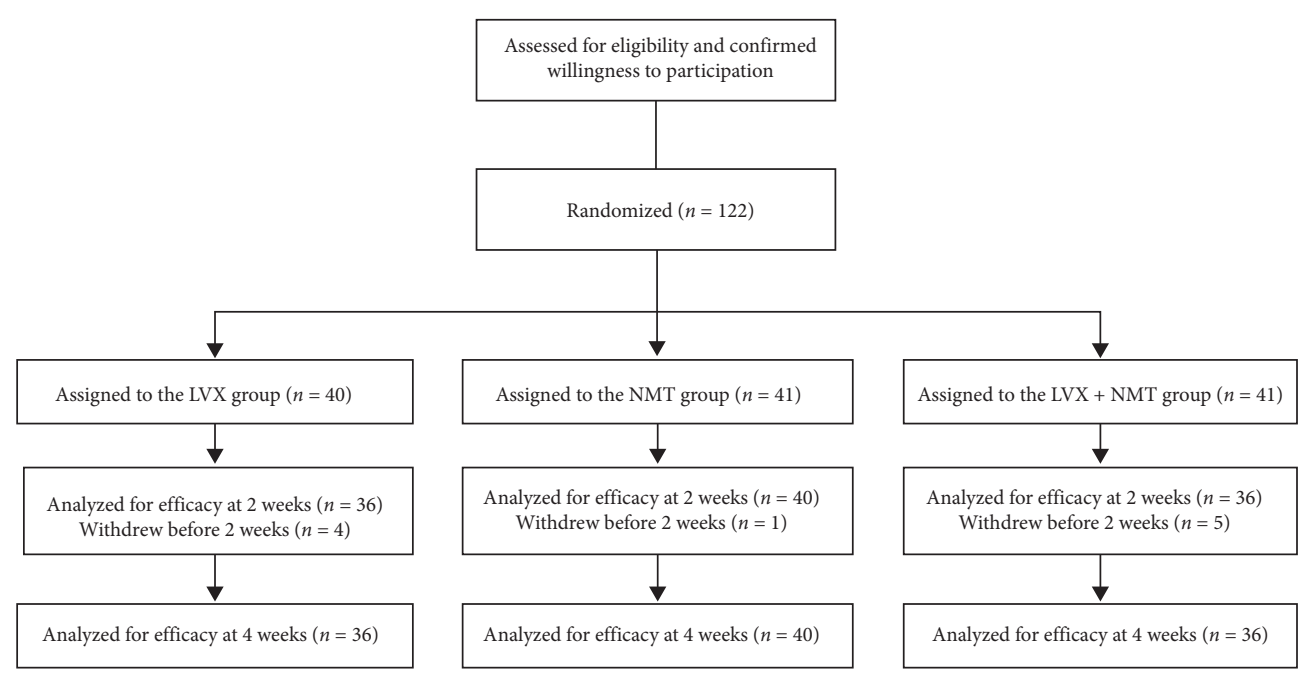

Figure 1: Patient flowchart.

TABle 1: Patient demographics and characteristics at baseline (mean $\pm \mathrm{SD}$ ).

\begin{tabular}{|c|c|c|c|c|c|c|c|}
\hline \multirow{2}{*}{ Variable } & \multicolumn{2}{|c|}{$\operatorname{LVX}(n=36)$} & \multicolumn{2}{|c|}{ NMT $(n=40)$} & \multicolumn{2}{|c|}{$\mathrm{LVX}+\mathrm{NMT}(n=36)$} & \multirow[t]{2}{*}{$P$ value } \\
\hline & Mean \pm SD & Range & Mean \pm SD & Range & Mean \pm SD & Range & \\
\hline Age (years) & $36.00 \pm 9.51$ & $20 \sim 58$ & $41.28 \pm 8.51$ & $25 \sim 59$ & $40.92 \pm 9.53$ & $25 \sim 58$ & 0.7796 \\
\hline CESI & $15.94 \pm 1.87$ & $12 \sim 21$ & $14.53 \pm 2.97$ & $8 \sim 21$ & $15.78 \pm 2.26$ & $13 \sim 23$ & 0.0520 \\
\hline $\operatorname{MDE}(\mathrm{cm})$ & $1.34 \pm 0.16$ & $0.95 \sim 1.60$ & $1.30 \pm 0.20$ & $0.94 \sim 1.72$ & $1.28 \pm 0.17$ & $0.90 \sim 1.67$ & 0.3702 \\
\hline
\end{tabular}

LVX, levofloxacin; NMT, Ningmitai; CESI, Chronic Epididymitis Symptom Index; MDE, mean diameter of epididymal nodules.

TABLE 2: CESI score after 2 or 4 weeks of treatment (mean \pm SD).

\begin{tabular}{lccc}
\hline Groups & LVX $(n=36)$ & NMT $(n=40)$ & LVX+ NMT $(n=36)$ \\
\hline CESI scores & & & $15.78 \pm 2.26$ \\
Baseline & $15.94 \pm 1.87$ & $14.53 \pm 2.97$ & $12.53 \pm 2.64^{* * * *}$ \\
2 weeks & $14.92 \pm 1.66$ & $13.33 \pm 3.22^{*}$ & $8.64 \pm 2.15^{* *, \# \#}$ \\
4 weeks & $10.39 \pm 2.31$ & $10.65 \pm 3.49$ & \\
\hline
\end{tabular}

LVX, levofloxacin; NMT, Ningmitai. ${ }^{*}, P<0.05$ compared with the LVX group. ${ }^{* *}, P<0.01$ compared with the LVX group. ${ }^{* * * *}, P<0.0001$ compared with the LVX group. ${ }^{\# \#} P<0.01$ compared with the NMT group.

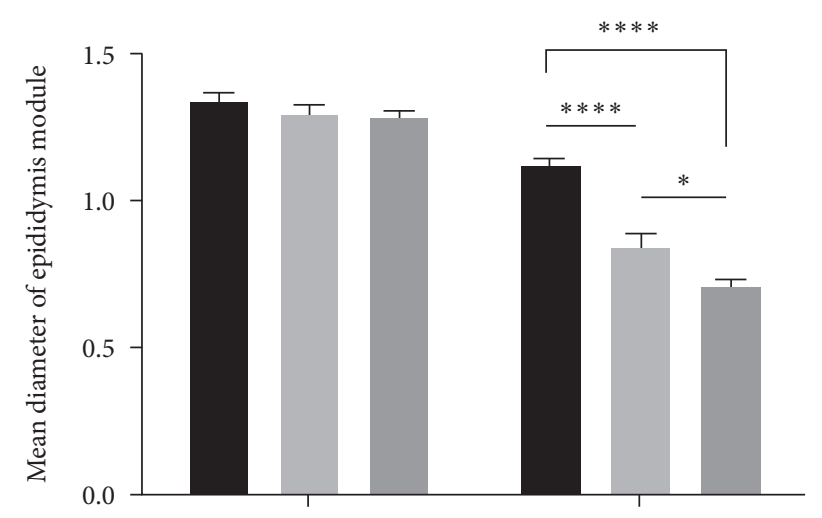

Baseline $\quad 4$ weeks

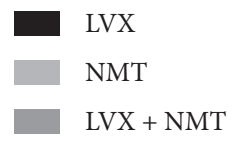

FIGURE 2: Mean diameter of epididymal nodules in the LVX group $(n=36)$, the NMT group $(n=40)$, and the combined group $(n=36)$ before and after 4 weeks of treatment. Unit, $\mathrm{cm} .{ }^{*} P<0.05^{* * * *}: P<0.0001$. LVX, levofloxacin; NMT, Ningmitai. 
TABLE 3: Clinical efficacy rates after 2 and 4 weeks of treatment.

\begin{tabular}{|c|c|c|c|c|c|c|}
\hline & \multicolumn{3}{|c|}{2 weeks } & \multicolumn{3}{|c|}{4 weeks } \\
\hline & LVX & NMT & LVX + NMT & LVX & NMT & $\mathrm{LVX}+\mathrm{NMT}$ \\
\hline$n$ & 36 & 40 & 36 & 36 & 40 & 36 \\
\hline \multicolumn{7}{|c|}{ Clinical efficacy, no. (\%) } \\
\hline Effective & $3(8.33)$ & $22(55.00)$ & $22(61.11)$ & $30(83.33)$ & $32(80.00)$ & $35(97.22)$ \\
\hline Ineffective & $33(91.67)$ & $18(45.00)$ & $14(38.89)$ & $6(16.67)$ & $8(20.00)$ & $1(2.78)$ \\
\hline$\chi^{2 \triangle}$ & - & 18.69 & 22.12 & - & 0.1401 & 2.5320 \\
\hline$P$ value $^{\triangle}$ & - & $<0.0001$ & $<0.0001$ & - & 0.7082 & 0.1116 \\
\hline
\end{tabular}

LVX, levofloxacin; NMT, Ningmitai. ${ }^{\triangle}$ Compared with the LVX group.

TABLE 4: Evaluation of hepatorenal function after 4 weeks of treatment.

\begin{tabular}{lcccc}
\hline & LVX & NMT & LVX+ NMT & Reference range \\
\hline$n$ & 36 & 40 & 36 & \\
ALT $\left(\mathrm{U} \cdot \mathrm{L}^{-1}\right)$ & & & $32.72 \pm 11.609$ & \\
$\quad$ V0 & $29.94 \pm 13.667$ & $28.33 \pm 10.406$ & $32.94 \pm 10.559$ & \\
$\quad$ V2 & $32.33 \pm 12.205$ & $31.88 \pm 8.762$ & & \\
BUN $\left(\mathrm{mmol} \cdot \mathrm{L}^{-1}\right)$ & $4.78 \pm 1.104$ & $4.97 \pm 0.933$ & $5.43 \pm 1.072$ & \\
$\quad$ V0 & $4.77 \pm 1.072$ & $5.13 \pm 1.145$ & $5.46 \pm 1.065$ & \\
V2 & & & & \\
CREA $\left(\mu \mathrm{mol} \cdot \mathrm{L}^{-1}\right)$ & $71.39 \pm 20.080$ & $69.50 \pm 20.796$ & $78.72 \pm 20.928$ & \\
V0 & $72.03 \pm 21.856$ & $61.30 \pm 17.164$ & $81.98 \pm 19.655$ & \\
V2 & & & \\
\hline
\end{tabular}

LVX, levofloxacin; NMT, Ningmitai; ALT, alanine aminotransferase; BUN, blood urea nitrogen; CREA, creatinine.

with LVX in the treatment of chronic epididymitis. Therefore, the efficacy of NMT in the treatment of chronic epididymitis needs further verification with larger scale, multicentre randomized controlled trials. Additionally, the pharmacological mechanism of action of NMT remains to be further explored via biomolecular experiments.

In conclusion, this parallel randomized controlled clinical study showed that NMT could significantly improve CESI scores and epididymal nodule diameters in patients with chronic epididymitis. The combination of NMT and LVX provided a better effect than monotherapy and was well tolerated.

\section{Data Availability}

The data used to support the findings of this study are included within the article.

\section{Disclosure}

Jing Zhang and Liying Guan are considered as the co-first authors.

\section{Conflicts of Interest}

The authors declare that there are no conflicts of interest.

\section{Authors' Contributions}

JZ and LYG collected data and drafted the manuscript. HZ was responsible for screening the patients for the study. ZQW, SL, and DQS evaluated patients and recorded data. $\mathrm{QF}$ and KQZ conceived the study, participated in its design, and gave final approval of the version to be published. All authors read and approved the final manuscript. Jing Zhang and Liying Guan contributed equally to this work.

\section{Acknowledgments}

This work was supported by the National Natural Science Foundation of China (81873830), the Natural Science Foundation of Shandong Province (ZR2017BH036), the Key Research Development Program of Shandong Province (2018GSF118142), the Shandong Provincial Key Research Program, China (2018GSF118083), and the Shandong Provincial Natural Science Foundation, China (ZR2019MH036).

\section{References}

[1] J. Wang, "The safety and efficacy of acupuncture for epididymitis protocol for a systematic review," Medicine (Baltimore), vol. 98, no. 1, Article ID e13934, 2019.

[2] L. D. Barbosa, M. Belotto, and R. D. Peixoto, "Epididymitis following cytoreductive surgery with intraperitoneal oxaliplatin chemotherapy: two case reports," Case Reports in Oncology, vol. 9, no. 1, pp. 138-142, 2016.

[3] S. Chen, H. Sun, H. Xu, Y. Zhang, and H. Wang, "Effects of acupuncture on hospitalized patients with urinary retention," Evidence-Based Complementary and Alternative Medicine, vol. 2020, Article ID 2520483, 7 pages, 2020.

[4] M. K. Kim, J. Leem, Y. I. Kim et al., "Gyejigachulbutang (GuiZhi-Jia-Shu-Fu-Tang, Keishikajutsubuto, TJ-18) in degenerative knee osteoarthritis patients: lessons and responders from a multicenter randomized placebo-controlled double-blind clinical trial," Evidence-Based Complementary and Alternative Medicine, vol. 2020, Article ID 2376581, 11 pages, 2020. 
[5] A. Shakeri, M. H. Hashempur, M. Mojibian, F. Aliasl, S. Bioos, and F. Nejatbakhsh, "A comparative study of ranitidine and quince (Cydonia oblonga mill) sauce on gastroesophageal reflux disease (GERD) in pregnancy: a randomised, openlabel, active-controlled clinical trial," Journal of Obstetrics and Gynaecology, vol. 38, no. 7, pp. 899-905, 2018.

[6] B. Ma, X. Chen, Y. Liang et al., "Efficacy of bloodletting therapy in patients with chronic idiopathic urticaria: a randomized control trial," Evidence-Based Complementary and Alternative Medicine, vol. 2020, Article ID 6598708, 11 pages, 2020.

[7] A. Khiveh, M. H. Hashempur, M. Shakiba et al., "Effects of rhubarb (Rheum ribes L.) syrup on dysenteric diarrhea in children: a randomized, double-blind, placebo-controlled trial," Journal of Integrative Medicine, vol. 15, no. 5, pp. 365-372, 2017.

[8] X. Yu and Q. Gao, "Chinese expert consensus on clinical application of Ningmitai capsule in chronic prostatitis," National Journal of Andrology, vol. 23, pp. 852-855, 2017.

[9] X. Chen, L. Zheng, and D. Chen, "Study on the inhibitory effect of Ningmitai capsule on the formation of staphylococcus, Escherichia coli and their biofilms in vitro," Chinese Journal of Andrology, vol. 26, pp. 552-558, 2020.

[10] H. Chen, Y. Xie, C. Deng et al., "The anti-inflammatory and antioxidative effects of Ningmitai capsule in the experimental autoimmune prostatitis rat model," Evidence-Based Complementary and Alternative Medicine, vol. 2020, Article ID 5847806, 7 pages, 2020.

[11] C. Jin, Z. Chen, and J. Zhang, "Meta-analysis of the efficacy of Ningmitai capsule on the treatment of chronic prostatitis in China," Medicine (Baltimore), vol. 97, no. 33, Article ID e11840, 2018.

[12] L. Liu, M. Q. Tian, and W. J. Huang, "Clinical observation of Ningmitai capsule in treating diabetic urinary tract infection," Chinese Journal of Integrated Traditional and Western Medicine in Intensive and Critical Care, vol. 04, pp. 368-371, 2014.

[13] K. Zhang, "Efficacy and safety of Ningmitai capsule in patients with chronic prostatitis/chronic pelvic pain syndrome: a multicenter, randomized, double-blind, placebo-controlled trial," Urology, 2021.

[14] B. Hu, D. Zheng, and T. Liu, "Clinical experience of Ningmitai capsule in treating 97 cases of acute epididymitis," Biotech World, vol. 10, p. 77, 2013.

[15] J. C. Nickel, D. R. Siemens, K. R. Nickel, and J. Downey, “The patient with chronic epididymitis: characterization of an enigmatic syndrome," Journal of Urology, vol. 167, no. 4, pp. 1701-1704, 2002.

[16] J. C. Nickel, "Chronic epididymitis: a practical approach to understanding and managing a difficult urologic enigma," Reviews in Urology, vol. 5, no. 4, pp. 209-215, 2003.

[17] J. C. Nickel, J. M. H. Teichman, M. Gregoire, J. Clark, and J. Downey, "Prevalence, diagnosis, characterization, and treatment of prostatitis, interstitial cystitis, and epididymitis in outpatient urological practice: the Canadian PIE study," Urology, vol. 66, no. 5, pp. 935-940, 2005.

[18] R. T. Strebel, C. Schmidt, J. Beatrice, and T. Sulser, "Chronic scrotal pain syndrome (CSPS): the widespread use of antibiotics is not justified," Andrology, vol. 1, no. 1, pp. 155-159, 2013.

[19] A. Khambati, S. Lau, A. Gordon, and K. A. Jarvi, "Onabotulinumtoxin A (botox) nerve blocks provide durable pain relief for men with chronic scrotal pain: a pilot open-label trial," The Journal of Sexual Medicine, vol. 11, no. 12, pp. 3072-3077, 2014.
[20] J. H. Chung, H. S. Moon, H. Y. Choi et al., "Inhibition of adhesion and fibrosis improves the outcome of epididymectomy as a treatment for chronic epididymitis: a multicenter, randomized controlled, single-blind study," Journal of Urology, vol. 189, no. 5, pp. 1730-1734, 2013.

[21] T. T. Carvalho, S. S. Mizokami, C. R. Ferraz et al., "The granulopoietic cytokine granulocyte colony-stimulating factor (G-CSF) induces pain: analgesia by rutin," Inflammopharmacology, vol. 27, no. 6, pp. 1285-1296, 2019.

[22] J. R. McConaghy and B. Panchal, "Epididymitis: an overview," American Family Physician, vol. 94, no. 9, pp. 723-726, 2016.

[23] Y. Lei, "Traditional Chinese medicine on treating epididymitis: a systematic review and meta-analysis protocol," Medicine (Baltimore), vol. 98, no. 24, Article ID e15975, 2019.

[24] N. A. Al Zahrani, R. M. El-Shishtawy, and A. M. Asiri, "Recent developments of gallic acid derivatives and their hybrids in medicinal chemistry: a review," European Journal of Medicinal Chemistry, vol. 204, Article ID 112609, 2020.

[25] G. Carullo, A. R. Cappello, L. Frattaruolo, M. Badolato, B. Armentano, and F. Aiello, "Quercetin and derivatives: useful tools in inflammation and pain management," Future Medicinal Chemistry, vol. 9, no. 1, pp. 79-93, 2017.

[26] L.-Q. Meng, F.-Y. Yang, M.-S. Wang et al., "Quercetin protects against chronic prostatitis in rat model through NF- $\kappa \mathrm{B}$ and MAPK signaling pathways," The Prostate, vol. 78, no. 11, pp. 790-800, 2018.

[27] X.-f. Shen, L.-b. Ren, Y. Teng et al., "Luteolin decreases the attachment, invasion and cytotoxicity of UPEC in bladder epithelial cells and inhibits UPEC biofilm formation," Food and Chemical Toxicology, vol. 72, pp. 204-211, 2014.

[28] Q. Yang, "Curative efficacy of Ningmitai capsules unite Tamsulosin hydrochloride sustained realeased capsules treatment of III type B prostatitis and its effects on level of the prostate fluid of IL-10, TNF- $\alpha$, PGE-2," Chinse Archives of Traditional Chinses Medicine, vol. 36, pp. 2750-2753, 2018.

[29] G. Liu and T. Ni, "Inhibitory effect of Ningmitai capsule on Staphylococcus epidermidis biofilm," Chinese Journal of Surgery of Integrated Traditional and Western Medicine, vol. 23, pp. 638-642, 2017. 